\title{
Can Functional Assessment Tools Reflect Balance Abilities at 3 Months after Total hip Arthroplasty?
}

\author{
Min-Woo Kim, MS, PT • Young-Uk Ryu, PhD, PT, $\mathrm{CSCS}^{1 \dagger}$
}

Department of Rehabilitation Medicine, Dongsan Medical Center, Keimyung University

${ }^{1}$ Department of Physical Therapy, Daegu Catholic University

Received: July 30, 2018 / Revised: August 1, 2018 / Accepted: August 23, 2018

(C) 2018 J Korean Soc Phys Med

\section{| Abstract |}

PURPOSE: This study was conducted to determine if the Harrison hip score (HHS), a tool for assessing hip joint function, and the Burg balance scale (BBS), a general balance assessment tool, actually reflect the balance ability of total hip arthroplasty (THA) patients 3 months after surgery. In addition, this study investigated the initial weight distribution strategy for bilateral lower extremity after THA surgery to understand the balance of THA patients.

METHODS: Fourteen 3-month THA patients performed static dual standing and sit-to-stand (STS) tasks. Ground reaction forces on each leg were collected to calculate the weight distribution symmetricity (SWD), and the HHS, functional HHS (f-HHS), and BBS were evaluated. Correlation analyses between SWD and the HHS (also f-HHS) and BBS were then applied to the THA patients.

RESULTS: The correlations between functional evaluation

$\dagger$ Corresponding Author: Young-Uk Ryu

ryuyounguk@gmail.com, http://orcid.org/0000-0003-1601-4477

This is an Open Access article distributed under the terms of the Creative Commons Attribution Non-Commercial License (http://creativecommons.org/licenses/by-nc/3.0) which permits unrestricted non-commercial use, distribution, and reproduction in any medium, provided the original work is properly cited. tools (HHS, f-HHS, BBS) and SWD were weak strength for the static balance task, but moderate for the dynamic STS task. Among the evaluation tools used in the present study, f-HHS was most useful for evaluation of dynamic balance ability. CONCLUSION: The results suggest that use of HHS, $\mathrm{f}-\mathrm{HHS}$, and BBS as functional evaluation tools does not provide meaningful information regarding balance ability, but that they are useful for evaluating dynamic balance ability of THA patients. The dynamic balance ability at 3 months after THA seems to be under development.

Key Words: Balance, Quiet standing, Sit-to-stand, Total hip arthroplasty

\section{Introduction}

Total hip arthroplasty (THA) is a common procedure used to replace the acetabulum and femoral head with artificial joints (Sanders et al., 2017). THA is used for pain relief and functional recovery from a wide range of hip joint diseases including avascular necrosis of the femoral head, degenerative and rheumatoid arthritis, and hip fractures (Bozic et al., 2009).

Many studies have shown that THA is effective at improving hip function (Sanei et al., 2016; Uhrbrand et al., 2014). However, in the case of balance ability, there 
have been conflicting reports depending on studies. Guralnik et al. (1995) reported a significant improvement in static balance at 12 months after THA. However, other studies have shown that the balance ability of THA patients shows slow improvement after the procedure (Majewski et al., 2005; Nallegowda et al., 2003). These findings indicate that assessing balance ability during rehabilitation of THA patients may be more important than evaluation of pain and function.

Thus, assessing balance ability can be an important index for examination of the functional recovery of patients following hip arthroplasty. Nevertheless, there are no specific balance assessment tools designed solely for patients with hip surgery. Instead, clinical use of general functional evaluation tools such as the Harrison hip score, Hip outcome score, or Oxford hip score (Tijssen et al., 2011), or general balance evaluation tools such as the Burg balance test are used to examine balance ability. However, few studies have examined how these evaluation tools reflect the balance ability of patients following hip surgery.

The most commonly used evaluation for effective clinical evaluation to represent the results of current hip surgery is the Harris hip score (HHS), which evaluates pain, function, and activity levels of hip surgery patients with high validity and reliability (interrater=.74 - 1.0) (Söderman and Malchau, 2001; Kirmit et al., 2005; Nilsdotter and Bremander, 2011). In recent years, the modified functional HHS (f-HHS) has been used, and evaluations using only functional items have been attempted (Reuling et al., 2012). Many studies of THA patients have also been used to evaluate hip joints (Aminian et al., 2004; Blomfeldt et al., 2007). The other balance evaluation tool that has been used for patients with hip surgery is the Berg balance scale (BBS) (Jogi et al., 2017). The BBS, which was originally created for the elderly to assess their balance, evaluates various behaviors related to static and dynamic balance and shows high validity and reliability (interrater=.98, intrarater=.97) (Berg et al., 1995).
This scale has also been used to assess the balance of patients with THA (Berg et al., 1992; Blum and Korner-Bitensky, 2008), as well as that of patients with multiple balance problems (Trudelle-Jackson and Smith. 2004).

Although the HHS and BBS have been used to evaluate hip joint function and balance in THA patients, it is unclear whether these two assessment tools actually reflect the balance of THA patients. Therefore, this study was conducted to investigate whether HHS, a tool for assessing hip joint function, and BBS, a general balance assessment tool, actually reflect the balance ability of THA patients. To accomplish this, BBS and HHS (and f-HHS) were analyzed through correlation analysis based on the measured balance ability of weight distribution characteristics during static and dynamic balance tasks.

\section{Methods}

\section{Participants}

This study was approved by the Medical Research Ethics Review Board (IRB) of Dongsan Medical Center, Keimyung University (IRB\#: 11-89). Fourteen patients who participated in total hip arthroplasty in the hospital were included and voluntarily participated in the study. The inclusion criteria were as follows: the ability to sit and walk independently; no other disease that could affect hip function; no cognitive impairment to understanding and following instructions. The general characteristics of the participants are shown in Table 1.

\section{Procedures}

Surgery was performed while the patient was lying on their side under general anesthesia, and all skin incisions were performed using an anterolateral approach. Only one side of hip joints were treated, and all patients underwent cementless procedures after capsular resection. The artificial hip joints used for the participants were the CLS 
Table 1. General Characteristics of Participants

\begin{tabular}{|c|c|c|}
\hline \multicolumn{2}{|c|}{ General characteristics } & Number \\
\hline \multirow{2}{*}{ Gender } & Male & 6 \\
\hline & Female & 8 \\
\hline \multirow{4}{*}{ Age (years) } & $40-49$ & 1 \\
\hline & $50-59$ & 3 \\
\hline & $60-69$ & 5 \\
\hline & $70-79$ & 5 \\
\hline \multirow{3}{*}{ Indication for surgery } & AVN & 4 \\
\hline & $\mathrm{OA}$ & 6 \\
\hline & Fx. & 4 \\
\hline \multirow{2}{*}{ Operated side } & Right & 6 \\
\hline & Left & 8 \\
\hline \multirow{5}{*}{ Time since surgery (day) } & $70-80$ & 3 \\
\hline & $80-90$ & 4 \\
\hline & $90-100$ & 3 \\
\hline & $100-110$ & 2 \\
\hline & $110-120$ & 2 \\
\hline
\end{tabular}

AVN: avascular necrosis of femoral head.

OA: osteoarthritis. Fx.: fracture.

the Spotorno ${ }^{\circledR}$ Stem, Trilogy ${ }^{\circledR}$ Acetabular Cup, DuPuy ${ }^{\circledR}$ SUMMIT $^{\circledR}$ Tapered Hip System, and Pinnacle ${ }^{\circledR}$ Acetabular Cup. From the second day after operation, quadriceps and joint exercises were started, and from days 2 to 3, gait training with crutches or walkers was started with partial weight bearing.

The research procedure was performed at three months after THA. Weight loadings on both legs were measured during quiet standing and sit-to-stand (STS) exercises. In addition, the participants were evaluated for BBS and HHS (including f-HHS). Two force plates (AMTI, Newton, MA, USA) were used to measure ground reaction forces (GRF) distributed on each leg. Data were collected at a sampling frequency of $200 \mathrm{~Hz}$ and the analog data generated from the force plates was converted to digital using an $\mathrm{A} / \mathrm{D}$ converter and stored on the hard disk of the computer.
During quiet standing, the participants were barefoot and stood comfortably on each force plate with their feet widened. During the test, they gazed naturally toward the front with their arms in a relaxed position next to the torso for 30 seconds. Three replicates were collected and three minutes of rest were given between measurements. After quiet standing, weight loadings for STS were measured. During this test, the participant sat on a chair with both feet on each force plate and then stood up. An adjustable height chair with no backrest was used, and the height of the chair was such that the knee angle was $90^{\circ}$ in the sitting position. During the STS, the participant was placed in a natural position with their hands next to the torso while gazing toward the front. The STS was repeated three times, with a rest of 3 minutes between measurements.

At 10-minutes after the STS, the BBS was evaluated. The BBS consisted of three areas: sitting, standing, and posture change. The total of the 14 items was 56 points based on individual scores of 0 to 4 . The items scored were sitting to standing, standing unsupported, sitting unsupported, standing to sitting, transfers, standing with eyes closed, standing with feet together, reaching forward with arms outstretched, retrieving an object from the floor, turning to look behind, turning 360 degrees, placing alternate foot on the stool, standing with one foot in front, and standing on one foot.

The HHS, which consists of questions and physical assessments, was evaluated after BBS evaluation. In the HHS, questions are categorized into three items: pain (0-44 points), function (0-47 points), and activity level. Additionally, physical assessment was evaluated based on the degree of hip motion, with the scores of individual items summed and the highest score being 100 points. Final scores were ranked as excellent $(\geq 90)$, good $(90-80)$, fair $(80-70)$, poor $(70-60)$, and failure $(<60)$. The evaluation of the functional parts of the HHS (f-HHS) was divided into walking (33 points) and specialized activities (14 points). Items related to walking consisted of limp (11 
Table 2. Descriptive Statistics of Each Variable

\begin{tabular}{cccc}
\hline & Mean & Min-Max & St. dev. \\
\hline BBS (max 56) & 50.79 & $43-56$ & 4.37 \\
HHS (max 100) & 81.14 & $61-98$ & 9.85 \\
f-HHS (max 47) & 32.57 & $26-45$ & 6.45 \\
SWDstand & .75 & $.39-.20$ & .18 \\
SWDsts & .66 & $.48-.80$ & .08 \\
\hline
\end{tabular}

BBS: Burg balance scale, HHS: Harrison hip score, f-HHS: functional Harrison hip score, SWD stand: symmetricity of weight distribution in quiet standing, SWDsts: symmetricity of weight distribution in sit-to-stand

points), use of walking aids (11 points) and walking distance (11 points), while items related to various specialized activities included stairs (4 points), shoes and socks (4 points), sitting (5 points), and public transportation (1 point).

The time spent evaluating the 2 assessment tools and collecting the balance data was about one hour per subject. All research procedures were performed by a skilled physical therapist to ensure reliability.

\section{Data analysis and statistics}

The GRFs of both feet collected from each force plate were used to calculate the symmetricity of weight distribution (SWD), which is a measure of the degree of body weight distribution imposed on each leg. After measuring the values of the GRF from both force plates, the mean value of the two GRFs was calculated from the mean of the three measurements. The SWD during quiet standing was calculated by dividing the average GRF on the operation side by the average GRF on the non-operation side. The SWD at STS was calculated by dividing the average peak GRF on the operation side by the average peak GRF on the non-operation side. When the SWD was 1 , the operation and non-operation sides represent a perfect 1:1 weight distribution between the two legs. An SWD higher than 1 indicates that more weight is applied to the operation side, while an SWD lower than 1 indicates that more weight is applied to the non-operation side.

Pearson correlation coefficients were used to evaluate the correlation between static and dynamic balance ability assessments and functional assessment tools (BBS, HHS, f-HHS). The significance level $(\alpha)$ for the statistical significance set at .05 .

\section{Results}

\section{Descriptive characteristics}

The mean, range, and standard deviation of each variable are shown in Table 2. The BBS ranged from 43 to 56 , meaning that participants' balance ability was good. The HHS ranged from 61 to 98 , reflecting participants with poor hip function to those with superior performance, respectively. The average SWD during quiet standing was .75 , but the range of values was large. These findings suggests that the participants maintained a static posture with different weight bearing strategies. The SWD in the STS was .66, but the range and standard deviation were less than those of the quiet standing, indicating that participants conducting STS used a similar strategy (with more weight on the non-operation leg) than those conducting quiet standing.

\section{Correlation between SWD and functional evaluations during quiet standing}

Weak correlation was found in the SWD in BBS and HHS of quiet standing (Table 3 ). 
Table 3. Correlation of BBS, HHS, f-HHS and SWD (SWDstand) in Quiet Standing

\begin{tabular}{cccc}
\hline & BBS & HHS & f-HHS \\
\hline SWDstand & $-.304 *$ & $-.347 *$ & -.184 \\
\hline
\end{tabular}

$* \mathrm{p}<.05$

BBS : Burg balance scale, HHS : Harrison hip score, f-HHS : functional Harrison hip score,

SWDstand : symmetricity of weight distribution in quiet standing

Table 4. Correlation of BBS, HHS, f-HHS and SWD (SWDsts) in sit-to-stand

\begin{tabular}{cccc}
\hline & BBS & HHS & f-HHS \\
\hline SWDsts & $.544 *$ & $.558 *$ & $.604 *$ \\
\hline
\end{tabular}

$* \mathrm{p}<.05$

BBS : Burg balance scale, HHS : Harrison hip score, f-HHS : functional Harrison hip score,

SWDsts : symmetricity of weight distribution in sit-to-stand

\section{Correlation between SWD and functional} evaluations in the STS

During the STS, the correlation between the SWD and the functional evaluation tools showed a positive trend with more than moderate correlation (Table 4). This indicates that a higher functional evaluation tools score was associated with more symmetrical weight bearing on both feet during the STS activity. Among the functional evaluation tools, the f-HHS showed the highest correlation, followed by the HHS and BBS (Table 4).

\section{Discussion}

This study examined whether static and dynamic balance capabilities of THA patients were reflected by functional assessment tools (BBS, HHS, and f-HHS). To accomplish this, we measured the balance ability and evaluated the hip joint function in patients approximately 3 months after THA. The results showed that there was a weak correlation between static balance and the evaluation tools, while there was a moderate correlation between dynamic balance ability and the tools. These findings suggest that the static balance ability of THA patients cannot be predicted based on the score of the functional evaluation tools, but that it can be predicted using the dynamic balance ability. Moreover, the f-HHS was the best predictor of dynamic balance capability. We will further discuss these results of the present study below.

First, there was no correlation or weak correlation between balance ability and hip joint function evaluation during quiet standing. Why do these tools fail to accurately reflect the ability of THA patients to maintain static posture? The ability to maintain static posture is an ability that is used throughout the lifetime that involves very basic balance ability (Seo et al., 2016). Since there are no other external forces acting during static posture maintenance, a minimum amount of energy is used when maintaining static posture relative to a different posture or dynamic posture (Houdijk et al., 2009). Therefore, it would not have been difficult for the participants to maintain a good posture by applying more weight to the non-operated leg, even though they had surgery on one hip. In addition, the results of this study indicate that the participants' SWD spread over a wide range, meaning that the patients maintained their static posture using a variety of strategies associated with double leg weight loads. That is, the participants maintained their static posture with their own weight-bearing strategies, regardless of balance ability or 
hip function. These results indicate that the initial static balance ability after THA surgery can be maintained regardless of the predicted balance and function evaluation tools. Nantel et al. (2008) examined the maintenance of static posture in patients with THA and found more postural fluctuation than in those with arthritis during quiet standing, indicating that it is not easy to control the static posture balance of THA patients. Talis et al. (2008) showed that THA patients had asymmetrical weight loading during quiet standing, but did not show as wide a range as was observed in the present study.

Unlike the results observed during quiet standing, all participants placed more weight on the non-operation side during the STS as a dynamic balance task. Recent studies also showed that the floor reaction force of the operative side of the THA patients was lower than that of the non-operated side during the STS of THA patients approximately 3 months postoperatively (Abujaber et al., 2015). These results indicate that the operated hip was still not clinically stabilized at 3 months postoperatively, indicating that the patients performed STS using force generated from the leg of the healthy side. The finding that the difference in SWD among participants was not greater than that for the static balance also showed that participants in this study used a similar strategy to conduct STS with more weight loading on the non-operation side. In the present study, the mean SWD in the dynamic activity (STS) of patients about 3 months after THA was .66, which is much lower than the ideal SWD of 1 , further indicating that the operated side had not been functionally restored. Boonstra et al. (2011) had 19 patients perform STS an average of 12.5 months after THA and found that the SWD was .83. These findings indicated that the functional burden of the bilateral lower limbs become similar when performing dynamic activities after more than one year compared to the 3-month weight symmetry observed in the present study. These findings indicate that the functional ability of the operated hip continuously improved with time after 3 months of THA.

In this study, even though the patients used the strategy of STS with more weight on the non-operation side, correlation analysis showed that a better balance and function score was associated with more weight support given to the operative hip. Thus, a better the balance and hip functional score was associated with more weight placed on the operative hip to maintain a dynamic balance. In other words, a higher the score of the evaluation tool used indicated more functional recovery of the operative hip. These results indicate that the balance and functional assessment tools fully reflect the function and recovery of the hip on the surgical side. As mentioned above, the $\mathrm{f}$-HHS is the best functional tool associated with dynamic balance ability because STS movement is more heavily influenced by hip joint function.

It should be noted that this study was limited in that the participants were evaluated about 3 months after THA surgery. Since THA surgery is performed for functional recovery of the hip joint, it is important to examine how the hip joint functions and the balance of THA patients is restored and developed through a longitudinal study rather than a particular time spot used in the present study. Another limitation of this study was that it included a limited number of cases. In future studies, more subjects should be recruited to generalize the present results.

\section{Conclusion}

The present study examined the correlation between balance abilities (static and dynamic) and functional assessment tools (HHS, f-HHS, and BBS) at 3 months after THA surgery. The results showed that the functional assessment tools were weakly correlated with static balance, but moderately correlated with dynamic balance at 3 months after THA. These findings suggest that static balance can be maintained with the patient's own weight-bearing strategies, regardless of balance ability or hip function, but 
that in dynamic balance tests such as the STS, more weight can be placed on the operative hip with better balance and hip functional score. When compared with previous studies (Abujaber et al., 2015; Boonstra et al., 2011), the dynamic balance ability at 3 months after THA surgery seems to be under development.

\section{References}

Abujaber SB, Marmon AR, Pozzi F, et al. Sit-to-stand Biomechanics before and after total hip Arthroplasty. J arthropl. 2015;30(11):2027-33.

Aminian K, Trevisan C, Najafi B, et al. Evaluation of an ambulatory system for gait analysis in hip osteoarthritis and after total hip replacement. Gait Posture. 2004;20(1):102-7.

Berg KO, Wood-Dauphinee SL, Williams J, et al. Measuring balance in the elderly: validation of an instrument. Can J Public Health. 1992;83(Suppl 2):7-11.

Berg KO, Wood-Dauphinee SL, Williams JI. The Balance Scale: reliability assessment with elderly residents and patients with an acute stroke. Scand J Rehabil Med. 1995;27(1):27-36.

Blomfeldt R, Törnkvist H, Eriksson K, et al. A randomised controlled trial comparing bipolar hemiarthroplasty with total hip replacement for displaced intracapsular fractures of the femoral neck in elderly patients. J Bone Joint Surg. 2007;89(2):160-5.

Blum L, Korner-Bitensky N. Usefulness of the Berg Balance Scale in stroke rehabilitation: a systematic review. Phys Ther. 2008;88(5):559-66.

Boonstra MC, Schreurs BW, Verdonschot N. The sit-to-stand movement: differences in performance between patients after primary total hip arthroplasty and revision total hip arthroplasty with acetabular bone impaction grafting. Phys Ther. 2011;91(4):547-54.

Bozic KJ, Kurtz SM, Lau E, et al. The epidemiology of revision total hip arthroplasty in the United States. J Bone
Joint Surg. 2009;91(1):128-33.

Guralnik JM, Ferrucci L, Simonsick EM, et al. Lower-extremity function in persons over the age of 70 years as a predictor of subsequent disability. N Engl J Med. 1995;332(9):556-62.

Houdijk H, Fickerta R, van Velzena J, et al. The energy cost for balance control during upright standing. Gait Posture. 2009;30(2):150-4.

Jogi P, Overend T, Kramer J. Comparisons of clinically based outcome measures and laboratory-based outcome measure for balance in patients following total hip and knee arthroplasty. Orthop Res Rev. 2017;9(1): 23-33.

Kirmit L, Karatosun V, Unver B, et al. The reliability of hip scoring systems for total hip arthroplasty candidates: assessment by physical therapists. Clin Rehabil. 2005;19(6):659-61.

Majewski M, Bischoff-Ferrari HA, Grüneberg C, et al. Improvements in balance after total hip replacement. J Bone Joint. 2005;87(10):1337-43.

Nallegowda M, Singh U, Bhan S, et al. Balance and gait in total hip replacement: a pilot study. Am J Phys Med Rehab. 2003;82(9):669-77.

Nantel J, Termoz N, Centomo H, et al. Postural balance during quiet standing in patients with total hip arthroplasty and surface replacement arthroplasty. Clin Biomech. 2008;23(4):402-7.

Nilsdotter A, Bremander A. Measures of hip function and symptoms: Harris Hip Score (HHS), Hip Disability and Osteoarthritis Outcome Score (HOOS), Oxford Hip Score (OHS), Lequesne Index of Severity for Osteoarthritis of the Hip (LISOH), and American Academy of Orthopedic Surgeons (AAOS) Hip and Knee Questionnaire. Arthritis Care Res. 2011; 63(S11):S200-7.

Reuling EM, Sierevelt IN, van den Bekerom MP, et al. Predictors of functional outcome following femoral neck fractures treated with an arthroplasty: limitations of 
the Harris hip score. Arch Orthop Trauma Surg. 2012;132(2):249-56.

Sanders TL, Kremers HM, Schleck CD, et al. Subsequent total joint arthroplasty after primary total knee or hip arthroplasty: a 40-year population-based study. J Bone Joint Surg. 2017;99(5):396-401.

Sanei F, Jamebozorgi AA, Irani A, et al. Comparing the quality of life before and after total hip arthroplasty operation in patients with developmental dysplasia of the hip. Phys Treat Spec Phys Ther J. 2016;5(4):219-24.

Seo SH, Bae HB, Cho YJ, et al. Immediate Effect of Eye Movement on Static Balance according to Age in Elderly Individuals. J Kor Phys Ther. 2016;28(5): 274-8.

Söderman P, Malchau H. Is the Harris hip score system useful to study the outcome of total hip replacement? Clin Orthop Relat Res. 2001;384(1):189-97.
Talis VL, Grishin AA, Solopova IA, et al. Asymmetric leg loading during sit-to-stand, walking and quiet standing in patients after unilateral total hip replacement surgery. Clin Biomech. 2008;23(4):424-33.

Tijssen M, van Cingel R, van Melick N, et al. Patient-Reported Outcome questionnaires for hip arthroscopy: a systematic review of the psychometric evidence. BMC musculoskeletal disorders. 2011;12(1):117-24.

Trudelle-Jackson E, Smith SS. Effects of a late-phase exercise program after total hip arthroplasty: a randomized controlled trial 1, 2. Arch Phys Med Rehab. 2004;85(7):1056-62.

Uhrbrand P, Ulrich M, Søballe K. Quality of life and hip function during the first month after total hip arthroplasty. Current Orthopaedic Practice. 2014; 25(3):233-7. 